www.jmscr.igmpublication.org

Index Copernicus Value: 79.54

ISSN (e)-2347-176x ISSN (p) 2455-0450

crossref DOI: https://dx.doi.org/10.18535/jmscr/v7i3.249

\title{
Effectiveness of costochondral joint mobilization with deep breathing in Tietze syndrome: A case study
}

\author{
Authors \\ Ruchi Mehta ${ }^{1^{*}}$, Priyanka Chaudhari ${ }^{2}$, Khatri $\mathrm{SM}^{3}$ \\ ${ }^{1}$ Assistant Professor, ${ }^{2}$ Tutor, ${ }^{3}$ Principal \\ Nootan College of Physiotherapy, Sankalchand Patel University, Visnagar, Gujarat, India 384315 \\ *Corresponding Author \\ Ruchi Mehta \\ Email: ruchi.ph@gmail.com
}

\begin{abstract}
Forthwith there is limited evidence about Tietze syndrome, its incidence/prevalence and effectiveness of physiotherapy interventions. We describe the effectiveness of costochondral joint mobilization with deep breathing and other passive physiotherapy interventions in a 27 year old male patient. Two weeks later the treatment protocol there was considerable reduction in his symptoms. Hence we propose that costochondral joint mobilization with deep breathing may be considered as an adjunct in the management of Tietze syndrome.
\end{abstract}

Keywords: Chest pain, Physiotherapy, Musculoskeletal system, Tietze syndrome.

\section{Introduction}

Chest pain is a common complaint in emergency departments, and the underlying pathology ranges from completely benign to imminently lifethreatening conditions. Among the innumerable possible etiologies are the confounding musculoskeletal disorders, which account for $50 \%$ of benign causes. While frequently discussed and included in differential diagnosis, they are often inappropriately misunderstood or mismanaged. ${ }^{1}$

Tietze syndrome became well known to the health care providers since 1921 when it was described for the first time by Alexander Tietze, a German surgeon. It is also known as costochondritis, chondropathia tuberose, anterior chest wall syndrome, costo sterna syndrome, parasternal chondrodynia, thoracochondalgia, and costochondral junction syndrome. It is believed to be one of the rare inflammatory disorders that presents with localized mild to severe anterior chest pain and tenderness in one or more of upper four ribs. $^{2,3}$

Exact prevalence of Tietze syndrome is unknown in India but in general it is considered as one of the uncommon muscusloekeltal disorder affecting anterior chest. There is a lacking of confirmatory diagnostic laboratory or imaging tests, thus clinical reliability of patient's history as well presentation is important. Most of the individuals who are diagnosed with this syndrome goes through medical evaluation and get referred for Physiotherapy treatment for the same ${ }^{4,5}$ 
Physical therapy is often considered one of the key management of Tietze syndrome since it is noninvasive, affordable, and convenient also helps patient to get involved in treatment that gives an opportunity for the individuals to have more contact time with health care specialist. ${ }^{6,7}$

Since there is limited information available about the evidence based guidelines for Physiotherapy management of costochondritis in general, we thought of reporting a young male patient with a typical case of Tietze's syndrome.

\section{Cases Study}

History: We describe a 27 year old male patient. His height was 162 centimeters, weight 58 kilograms and body mass index (BMI) was 22.1. The patient was seen by a female physiotherapist and enrolled for daily treatment. He was referred for physiotherapy by a general physician and diagnosed as a case of costochondritis. He complained of sharp stabbing, achy or pressure like pain over anterior chest pain on left side that got aggravated with deep inspiration, sneezing and coughing since fifteen days. He belonged to a middle socioeconomic class and fair family and social support. He had no history of strenuous activity, trauma or similar complaint in the past.

Physical examination: His vital signs were normal. Careful palpation of the anterior chest wall using a single digit reproduced his pain at left upper costochondral joints. Auscultatory findings were normal, rib and shoulder movements were normal. There was bilateral pectoral tightness and crowing rooster maneuver was positive. His radiographs were normal and MRI showed thickening, enlargement of cartilage at left upper three costochondral junctions.

Procedure: Ethical approval was granted from the Institutional Ethical Committee and the patient gave an informed written consent. His demographic data, physical examination, intensity of pain on visual analogue scale and global rating of change scale score was noted. He was treated with phonophoresis with piroxicam ointment and aquasonic gel with $0.25 \mathrm{w} / \mathrm{cm}^{2}$ for 3 minutes with
$1 \mathrm{MHz}$ pulsed mode ultrasound with 1:10 pulse ratio was applied at the site of pain. After this he received painless costochondral joint mobilization with deep breathing for 3 sets of 10 repetitions (Fig.1) and taping with elasto plast for three hours on alternate days for fifteen days. He was advised to take rest, avoid strenuous activity, avoid lifting heavy weights and apply hot water fomentation and local analgesic ointment. After fifteen days, his pain got reduce from 6.9 to 2.4 on visual analogue scale and also on patient-specific functional scale (PSFS) score got changed from 6.3 to 1.7 .

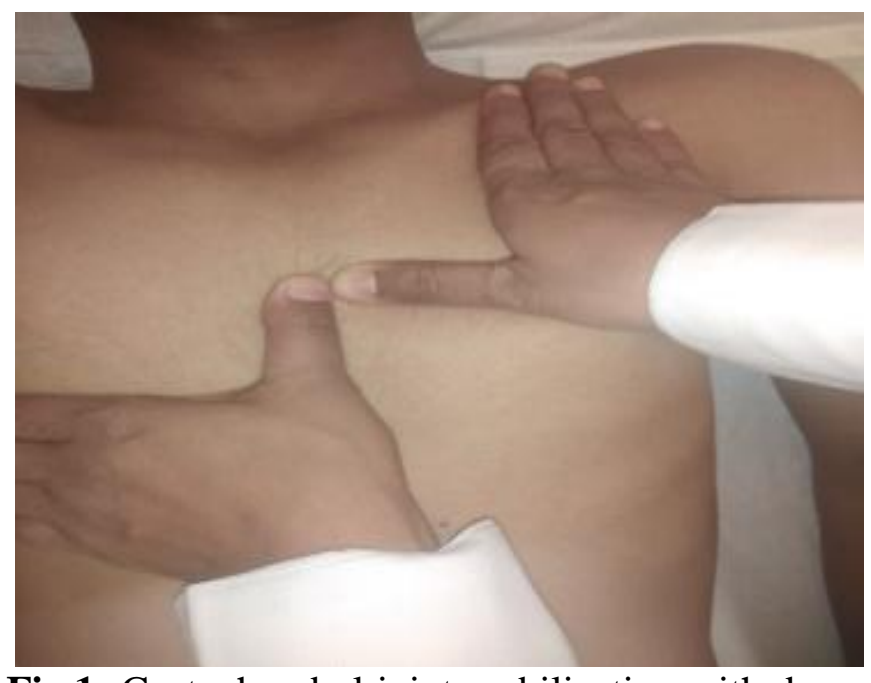

Fig.1: Costochondral joint mobilization with deep breathing

\section{Discussion}

This case study provides information about the effectiveness of costochondral joint mobilization with deep breathing in the management in the treatment of costochondritis. We found that this combined movement with mobilization was painless and clinically effective for our patient. This could be due to mechanical correction of rib dysfunction, neuro physiological, psychological or placebo effect. ${ }^{8}$ However, since additional interventions were used in the management of this patient so we believe that this could be a combined effect of physiotherapy interventions suggesting the clinical utility of such intervention as an adjunct in the treatment of costochondritis. However, we are aware about the issues of methodological rigor, researcher subjectivity, 
external validity of single subject research and would like to recommend similar research with a different kind of research design in future.

\section{Conclusion}

Costochondral joint mobilization with deep breathing can be used as an accompaniment in the treatment of Tietze syndrome.

\section{References}

1. Gijsbers, E. and Knaap, S.F. (2011) Clinical presentation and chiropractic treatment of Tietze Syndrome: A 34-yearold female with left-sided chest pain. Journal of Chiropractic Medicine, 10, 6063. doi:10.1016/j.jcm.2010.10.002.

2. https://rarediseases.org/rarediseases/tietze-syndrome/ accessed on 12 January 2019.

3. Anthony S. Fauci, Carol A. Langford. Harrison's rheumatology. Second edition. 2010. McGraw-Hill Education.

4. Hiramuro-Shoji, F., Wirth, M.A. and Rockwood Jr., C. (2003) Atraumatic conditions of the sternoclavicular joint. Journal of Shoulder and Elbow Surgery, 12, 78-88.

5. Wolf, E. and Stern, S. (1976) Costosternal Syndrome: Its frequency and importance in differential diagnosis of coronary heart disease. Archives of Internal Medicine, 136, 189-191.

6. Tietze, A. (1921) Uber eine eigenartige haufung von fallen mit dystrophie der rippenknorpel. Berliner Klinische Wochenschrift, 58, 829-831.

7. Proulx, A. Teresa. W.; Costochondritis: Diagnosis and Treatment; Am Fam Physician. 2009 Sep 15;80(6):617-620.

8. Wright A: Hypoalgesia post-manipulative therapy: a review of a potential neurophysiological mechanism. Manual Therapy: 1995(1):11-16. 